\title{
Using Discrete Wavelet Transform to Model Whistle Contours for Dolphin Species Classification ${ }^{+}$
}

\author{
Paula Lopez-Otero ${ }^{1, \ddagger * \mathbb{D}, \text { Laura Docio-Fernandez }}{ }^{2, \ddagger}$ and Antonio Cardenal-López ${ }^{2, \ddagger}$ \\ 1 Information Retrieval Lab, CITIC, Universidade da Coruña, 15071 A Coruña, Spain \\ 2 Multimedia Technology Group, atlanTTic Research Center, Universidade de Vigo, E36310 Vigo, Spain; \\ 1docio@gts.uvigo.es (L.D.-F.); cardenal@gts.uvigo.es (A.C.-L.) \\ * Correspondence: paula.lopez.otero@udc.gal; Tel.: +34-981-670000 (ext. 1276) \\ + Presented at the XoveTIC Congress, A Coruña, Spain, 27-28 September 2018. \\ $\ddagger$ These authors contributed equally to this work.
}

Published: 17 September 2018

\begin{abstract}
This work proposes the use of features based on the discrete wavelet transform (DWT) for dolphin species classification. These features are compared with other previously used in the literature, and the experiments carried out in a database featuring four different species of cetaceans (three dolphins and a pilot whale) showed that the use of DWT features led to improved classification performance.
\end{abstract}

Keywords: cetacean classification; whistle contour; discrete wavelet transform

\section{Introduction}

The health of marine mammal populations is often considered an indicator of overall marine ecosystem health and resilience [1], which makes it interesting to develop automatic tools to detect and classify cetacean species. Cetaceans produce characteristic sounds, such as whistles and clicks, that are used for different tasks such as navigation, communication or hunting [2]. The whistles are short narrow bandwidth sounds of short duration, and they consist in omnidirectional tones that vary with time, sometimes presenting a strong harmonic structure. In addition, whistle patterns vary between species due to physiological differences or environmental conditions, among others [3], making it possible to distinguish cetacean species using these sounds.

Several efforts for classifying cetacean species using whistle contours have been made. In [4] and [5], different parameters extracted from whistles were used to train a species classifier. Other features were explored in [6], such as the number of harmonics and frequency ratios. A statistical analysis of the discrimination capability of these features for classifying some dolphin species can be found in [7]. Other researchers used cepstral coefficients (CCs) for this task [8]. With respect to the classification strategy, some researchers rely on classifiers such as discriminant function analysis or classification and regression trees [3], while the use of Gaussian mixture models (GMMs) is also common [8,9]. In this work, a set of statistics obtained from the discrete wavelet transform of whistle contours is proposed for cetacean species classification.

\section{Materials and Methods}

In this work, four different cetacean species are considered: common dolphin Delphinus delphis (DDE), striped dolphin Stenella coeruleoalba (SCO), common bottlenose dolphin Tursiops truncatus (TTR) and long-finned pilot whale Globicephala melas (GME). A set of whistles was extracted as proposed in [9], which led to 24, 15, 15 and 23 whistles of DDE, GME, SCO and TTR, respectively. These whistles were extracted from a set of recordings collected by CEMMA (http:/ /www.cemma.org/) (Indemares 
Life 07/NAT/E/000732 project (http:/ /www.indemares.es)) in the Northwest of Spain (Galician Bank and Avilés Canyon) in 2010 and 2011.

The use of the discrete wavelet transform (DWT) for whistle characterization is proposed in this paper. The DWT is a multi-resolution technique that decomposes a time series into subsequences at different resolution scales providing data into high and low-frequency components. At high frequency, the wavelets can capture discontinuities, ruptures and singularities in the original data. At low frequency, the wavelet characterizes the coarse structure of the data to identify the long-term trends. Thus, the wavelet analysis allows to extract the hidden and significant temporal features of the original data. The first step consists in decomposing the original signal into approximation (CA) and detail (CD) coefficients by convolving the signal with a low-pass filter (LP) and a high-pass filter (HP), respectively. The low-pass filtered signal is the input for the next iteration level and so on. The approximation coefficients contain the general trend (the low-frequency components) of the signal, and the detail coefficients contain its local variations (the high-frequency components).

The analysis of whistle contours using DWT was carried out as follows: first the whistle contours were processed with a DWT (Daubechies-5 wavelet) and both approximation $(a)$ and detail $(d)$ coefficients were extracted from these raw contours and its logarithm version in four levels. Then, several features were obtained: percentage of energy of $a$ and $d$ coefficients in each level; relative wavelet energy and wavelet entropy; Shannon energy entropy and log-energy entropy of $a$ and $d$ in each level; RMS and standard deviation of $a$ and $d$ in each level; mean, standard deviation, skewness and kurtosis of the Teager-Kaiser Energy Operator (TKEO) [10] of $a$ and $d$ in each level.

\section{Results}

Four different systems were assessed:

- CC. Cepstral coefficients combined with a likelihood classifier based on GMMs as proposed in [8]. CCs were computed using 51 filters separated $500 \mathrm{~Hz}$ from each other, and uniformly distributed between $4 \mathrm{KHz}$ and $30 \mathrm{KHz}$. The features were extracted every $1 \mathrm{~ms}$ using a $7 \mathrm{~ms}$ window.

- Whistle countour. Contour estimation consists in estimating the exact frequency of each whistle, aiming at obtaining an accurate detection of all the whistles in a signal. The frequency of whistle contours can be extracted using the unpredictability measure described in [9]. As in the case of CCs, the classification stage using whistle contours can be carried out by means of maximum posterior probability computation, as in [9].

- Whistle contour statistics (similarly to [3]): beginning and end frequencies, minimum and maximum frequencies, duration, slope of the beginning and end sweeps, number of inflection points, number of steps, frequency range. Support vector machine classifier with Gaussian kernel was used to perform species classification.

- Discrete wavelet transform as described in Section 2.

Table 1 shows the results of cetacean classification using the aforementioned systems following a leave-one-out strategy (the reported accuracies were obtained with the optimal parameters of each classifier). The outstanding performance of CCs for this classification task was proven to be misleading since the features are capturing information relative to the channel: this was demonstrated by means of a location-independent experiment, where these features showed an accuracy of $33.33 \%$. In addition, an analysis of the confusion matrices of the different systems showed that DWT features were the only ones that achieved an accuracy above $50 \%$ for all classes.

Table 1. Accuracies obtained in the cetacean species classification experiment using four different systems.

\begin{tabular}{cc}
\hline Features & Accuracy \\
\hline CC & $86.59 \%$ \\
Whistle contour & $55.84 \%$ \\
Whistle contour statistics & $63.64 \%$ \\
Discrete wavelet transform & $68.83 \%$ \\
\hline
\end{tabular}




\section{Conclusions}

This paper proposed the use of features extracted from the discrete wavelet transform for cetacean species classification using whistle contours. A comparison of these features with other approaches found in the literature showed an absolute improvement of $13 \%$ with respect to using the frequencies of the whistle contours and of $5 \%$ with respect to using a set of statistics extracted from the whistle contours. Nevertheless, the overall classification accuracy was around $69 \%$, so there is still room for improvement.

Funding: This work has received financial support from (i) "Ministerio de Economía y Competitividad" of the Government of Spain and the European Regional Development Fund (ERDF) under the research projects TIN2015-64282-R and TEC2015-65345-P, (ii) Xunta de Galicia (projects GPC ED431B 2016/035 and GRC2014/024), and (iii) Xunta de Galicia-“Consellería de Cultura, Educación e Ordenación Universitaria" and the ERDF through the 2016-2019 accreditations ED431G/01 ("Centro singular de investigación de Galicia") and ED431G/04 ("Agrupación estratéxica consolidada").

Acknowledgments: The authors would like to thank CEMMA (Coordinadora para o Estudo dos Mamíferos Mariños) for making available all its underwater recordings and information on the studied marine mammals and their behaviour.

Conflicts of Interest: The authors declare no conflict of interest. The founding sponsors had no role in the design of the study; in the collection, analyses, or interpretation of data; in the writing of the manuscript, and in the decision to publish the results.

\section{References}

1. Frasier, K.; Roch, M.; Soldevilla, M.; Wiggins, S.; Garrison, L.; Hildebrand, J. Automated classification of dolphin echolocation click types from the Gulf of Mexico. PLoS Comput. Biol. 2017, 13, e1005823.

2. Au, W.; Popper, A.; Fay, R. Hearing by whales and dolphins. In Springer Handbook of Auditory Research; Springer: New York, NY, USA, 2000.

3. Oswald, J.; Barlow, J.; Norris, T. Acoustic identification of nine delphinid species in the eastern tropical Pacific Ocean. Mar. Mamm. Sci. 2003, 19, 20-37.

4. Oswald, J.; Rankin, S.; Barlow, J.; Lammers, M. A tool for real-time acoustic species identification of delphinid whistles. J. Acoust. Soc. Am. 2007, 122, 587-595.

5. López, B.D. Whistle characteristics in free-ranging bottlenose dolphins (Tursiops truncatus) in the Mediterranean Sea: Influence of behaviour. Mamm. Biol. Z. Säugetierkd. 2011, 76, 180-189.

6. Soto, A.B.; Marsh, H.; Everingham, Y.; Smith, J.; Parra, G.; Noad, M. Discriminating between the vocalizations of Indo-Pacific humpback and Australian snubfin dolphins in Queensland, Australia. J. Acoust. Soc. Am. 2014, 136, 930-938.

7. Gannier, A.; Fuchs, S.; Quèbre, P.; Oswald, J. Performance of a countour-based classification method for whistles of Mediterranean delphinids. Appl. Acoust. 2002, 71, 1063-1069.

8. Roch, M.; Soldevilla, M.; Burtenshaw, J.; Henderson, E.; Hildebrand, J. Gaussian mixture model classification of odontocetes in the Southern California Bight and the Gulf of California. J. Acoust. Soc. Am. 2007, 121, 1737-1748.

9. Parada, P.P.; Cardenal-López, A. Using Gaussian mixture models to detect and classify dolphin whistles and pulses. J. Acoust. Soc. Am. 2014, 135, 3371-3380.

10. Kaiser, J. Some Useful Properties of Teager's Energy Operators. In Proceedings of the 1993 IEEE International Conference on Acoustics, Speech, and Signal Processing, Minneapolis, MN, USA, 27-30 April 1993; Volume III, pp. 149-152.

(c) 2018 by the authors. Licensee MDPI, Basel, Switzerland. This article is an open access article distributed under the terms and conditions of the Creative Commons Attribution (CC BY) license (http://creativecommons.org/licenses/by/4.0/). 